INPLASY

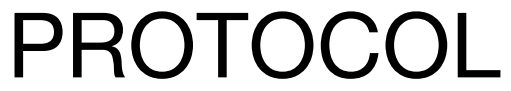

To cite: Martiny et al. Effects of flow states on elite athletes in invasion team sports:

systematic review protocol. Inplasy protocol 2021110114. doi:

10.37766/inplasy2021.11.0114

Received: 30 November 2021

Published: 30 November 2021

Corresponding author:

Luis Martiny

luis_martiny@hotmail.com

Author Affiliation:

Faculdade de Ciências do

Desporto e Educação Física -

Universidade de Coimbra.

Support: None.

Review Stage at time of this submission: Data analysis.

Conflicts of interest:

None declared.

\section{EFFECTS OF FLOW STATES ON ELITE ATHLETES IN INVASION TEAM SPORTS: SYSTEMATIC REVIEW PROTOCOL}

Martiny, L1; Theil, L2; Neto, E³; Dias, G4; Ferreira, P5; Mendes, R6.

Review question / Objective: How does the flow state occur in invasion team sports (e.g., prevents; disrupts; restores; triggers; facilitates; hampers) and how does it influence the Elite athletes performance?

Rationale: The flow state has been configured as an important flooring condition in the search for the best performance. However, there is an absence of systematizations about the flow state specifically in invasive team sports and its impact on performance. Therefore, it is relevant to qualify the types of studies conducted, their main correlations and causalities, as well as their main evidence and limitations.

Condition being studied: The flow in invasion team sports and its impact on the Elite athletes performance.

INPLASY registration number: This protocol was registered with the International Platform of Registered Systematic Review and Meta-Analysis Protocols (INPLASY) on 30 November 2021 and was last updated on 30 November 2021 (registration number INPLASY2021110114).

\section{INTRODUCTION}

Review question / Objective: How does the flow state occur in invasion team sports (e.g., prevents; disrupts; restores; triggers; facilitates; hampers) and how does it influence the Elite athletes performance?
Rationale: The flow state has been configured as an important flooring condition in the search for the best performance. However, there is an absence of systematizations about the flow state specifically in invasive team sports and its impact on performance. Therefore, it is relevant to qualify the types of studies conducted, their main correlations and 
causalities, as well as their main evidence and limitations.

Condition being studied: The flow in invasion team sports and its impact on the Elite athletes performance.

\section{METHODS}

Search strategy: Databases: Web of Science Core Colletion; SPORTDiscus (EBSCO-Host); PsycINFO e Pubmed Keywords: "flow" OR "flow state" OR "Dispositional flow" OR "The fundamentals of flow" OR "flow in sports" OR "Autotelic experience" OR "self-transcendence" OR "self*" OR "evolving self" OR "optimal experience" OR "Peak moment" OR "peak performance" OR "Peak experience" OR "IZOF" OR "in zone" OR "being in zone" OR "Mindfulness" OR "Meditation" OR "Entropy" OR "Genentropy" AND "team sport"” OR "team-sport" OR "hanball" OR "handebol" OR "Football" OR "Soccer" OR "Basketball" OR "Rugby" OR "futsal" OR "Hockey" OR "Ice Hockey" OR "water polo" AND "psychological" OR "mood" OR "emotion*" OR "Mental energy" OR "Mental" OR "Energy" OR "Spiritual" OR "Phisyological" OR "Technique" OR "Technical skill"” OR "Skill" OR "Tactical intelligence" OR "tactical" OR "decisionmaking in team-sport" " OR "decisionmaking" OR "cognitive performance" OR "cognitive*".

Participant or population: Elite athletes in invasion team sports.

Intervention: 1 Types of methodology used to improve, induce, facilitate, achieve flow2.1 mindfulness2.2 imagery meditation1.3 flow training1.4 Integral Yoga1.5 Self talk2 Types of teachinglearning-training methodology used2.1 Global method2.2 Teaching Games for Understanding (TGFU) or situational-active method2.3 Traditional method3. Types used to measure personality traits and nomothetic profile (emotion; energy; mood) associated (correlation or/and causality) with flow state3.1 Big five personality test3.2 Individual Zone of optimal functions (IZOF); 3.3 Hedonic orientation of emoticons experienced (Hedonic tone)3.4 Self-determination theory3.5 Dispositional flow state scale3.6 Satisfaction perception.

Comparator: Studies should try to correlate the flow state with some aspect of the athlete's performance, especially those that involve "cognitive performance" or with decision-making processes, whether through experimental, quasi-experimental, or cross-sectional research. They may also present correlations and / or casualties with moods, motivation, emotions and processes of teaching learning training.

Study designs to be included: 1) Original studies; experimental studies 2) Correlational OR/AND intervention studies.

Eligibility criteria: The inclusion criteria were: i) Only originals article ii) Only articles based on samples iii) Only articles made with team sports and flow iv) Language: English; Portuguese and Spanish v) Use of qualitative, quantitative or mix-methods vi) 2015-2020.

Information sources: The search was limited to original articles published online between 2015-2020. Literature reviews, overviews, conference proceedings, and masters and PhD. thesis.

Main outcome(s): To obtain a better understanding of the factors that affect the occurrence of the flow state in team sports; that is, to understand what are the limitations and the advantages of reaching the flow state in team sports. Especially the collective sports of invasion.

Additional outcome(s): A second focus of the systematic review is to understand the impact of the flow state on the athlete's performance, mainly with regard to their cognitive performance. More specifically, their decision-making process (time spent and quality in choosing the action taken). It consists in an attempt to map the consequences of the flow state. To this end, we will seek to identify the extent to which it is possible to measure and associate the combination of two important psychological constructs (flow states and 
intelligence - decision making process), based on the assumption that one affects the essentiality of the other.

Data management: Step 1 - Using the search strategies and keywords, the relevant titles of the articles selected in the search will be identified. A reference program will be used to handle this initial data. Step 2 - After the initial selection through the titles (which will be made by two members of the review team), the reading of the abstracts will begin. When reading the abstracts, the two team members will select those articles that will proceed to the full reading of the article, according to the eligibility criteria. If there are differences between the two reviewers, a third reviewer will be called. Step 3 -This is the phase of the complete reading of the articles. The reasons for excluding articles at this stage will be described in the PRISMA flow diagram (Moher, Liberati, Tetzlaff, \& Altman, 2009). Step 4 - Once the final sample of articles that will be part of the systematic review will be defined, the information coding phase will begin, which will include: a) the research identification data (study design; participants; author, year, country; type of team sports; intervention and measures; main outcomes) b) the results found (criteria that affect the state of flow in team sports and the effects of flow on the athlete's performance) and c) the final evaluation of the study.

Quality assessment / Risk of bias analysis: The PRISMA checklist was used (Galvão, Pansani, Harrad, 2015).

Strategy of data synthesis: A dashboard will be made for the extraction of information. This panel will include the following information: a) Study design; b) Identification data (First author, year and country); c) Participants (age, gender; expertise level); d) Team sports modalities; e) Intervention; f) Measures; g) Key findings. Main results will be divided into two categorie : i) affect to flow states in team sports and ii) performance effects (psychological (mood-emotions); physiological; technical tactical. h) assessment.

Subgroup analysis: Analysis of subgroups or subsets is not anticipated for this review.

Sensitivity analysis: None.

Language: English; Portuguese and Spanish.

Country(ies) involved: Brazil and Portugal.

Keywords: flow state; collective sports; athlete performance; metacognition; sport psychology.

Contributions of each author:

Author 1 - Luis Eugênio Martiny. Email: luis_martiny@hotmail.com Author 2 - Larissa Zanetti Theil. Email: larissatheil@gmail.com Author 3 - Eloy Maciel Neto. Author 4 - Gonçalo Dias. Author 5 - José Pedro Ferreira. Author 6 - Rui Mendes. 